\title{
IPSILATERAL BREAST TUMOR RELAPSE: LOCAL RECURRENCE VERSUS NEW PRIMARY TUMOR AND THE EFFECT OF WHOLE-BREAST RADIOTHERAPY ON THE RATE OF NEW PRIMARIES
}

\author{
Dorothy M. Gujral, M.R.C.P., ${ }^{*}$ Georges Sumo, M.Sc., ${ }^{\dagger}$ John R. Owen, F.R.C.R., ${ }^{\ddagger}$

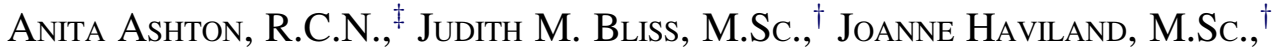 \\ AND JoHn R. YARNOLD, F.R.C.R.* \\ *Section of Academic Radiotherapy, Royal Marsden Hospital, Sutton, United Kingdom; ${ }^{\dagger}$ ICR-Clinical Trials and Statistics Unit, Section \\ of Clinical Trials, Institute of Cancer Research, Sutton, United Kingdom; and ${ }^{\ddagger}$ Department of Clinical Oncology, Gloucestershire \\ Oncology Centre, Cheltenham, United Kingdom
}

\begin{abstract}
Purpose: The justification for partial breast radiotherapy after breast conservation surgery assumes that ipsilateral breast tumor relapses (IBTR) outside the index quadrant are mostly new primary (NP) tumors that develop despite radiotherapy. We tested the hypothesis that whole-breast radiotherapy (WBRT) is ineffective in preventing NP by comparing development rates in irradiated and contralateral breasts after tumor excision and WBRT. Methods and Materials: We retrospectively reviewed 1,410 women with breast cancer who were entered into a prospective randomized trial of radiotherapy fractionation and monitored annually for ipsilateral breast tumor relapses (IBTR) and contralateral breast cancer (CLBC). Cases of IBTR were classified into local recurrence (LR) or NP tumors based on location and histology and were subdivided as definite or likely depending on clinical data. Rates of ipsilateral NP and CLBC were compared over a 15-year period of follow-up.

Results: At a median follow-up of 10.1 years, there were 150 documented cases of IBTR: 118 (79\%) cases were definite or likely LR; 27 (18\%) cases were definite or likely NP; and 5 (3\%) cases could not be classified. There were 71 cases of CLBC. The crude proportion of definite-plus-likely NP was $1.9 \%(27 / 1,410)$ patients compared with 5\% (71/1,410) CLBC patients. Cumulative incidence rates at 5, 10, and 15 years were $0.8 \%, 2.0 \%$, and $3.5 \%$, respectively, for definite-plus-likely NP and $2.4 \%, 5.8 \%$, and $7.9 \%$, respectively for CLBC, suggesting a difference in the rates of NP and CLBC.

Conclusions: This analysis suggests that WBRT reduces the rate of ipsilateral NP tumors. The late presentation of NP has implications for the reporting of trials that are testing partial breast radiotherapy. (C) 2011 Elsevier Inc.
\end{abstract}

Breast cancer, Ipsilateral, Contralateral, Relapse, Radiotherapy.

\section{INTRODUCTION}

The rationale for partial breast radiotherapy (PBRT) after breast conservation surgery for early breast cancer includes the assumption that most ipsilateral breast tumor relapses (IBTR) developing outside the index quadrant are new primary (NP) tumors that are not prevented by radiotherapy (1-3). Several phase I and II studies evaluating accelerated PBRT have reported promising early results, with few local tumor relapses, while maintaining excellent cosmetic outcomes and minimum toxicity (4-7). Currently, PBRT for early breast cancer is being investigated in several phase III randomized studies (8-12). However, evidence that whole- breast radiotherapy (WBRT) has little or no effect on the rate of ipsilateral NP tumors is not particularly robust, and a number of recent retrospective studies with long followup periods suggest a significant therapeutic effect (13-16). In order to test for such an effect, it is necessary to apply criteria that distinguish between local recurrences (LR) and NP tumors. The purpose of this study was to test the hypothesis that WBRT reduces the rate of ipsilateral NP, which otherwise occur at a rate comparable to that of contralateral breast cancer (CLBC). The research plan involved (i) classifying IBTR in breast cancer patients treated with breast-conserving therapy as LR or NP tumors based on location and histology of the
Note-An online CME test for this article can be taken at http:// asro.astro.org under Continuing Education.

Reprint requests to: Prof. John Yarnold, Section of Academic Radiotherapy, Royal Marsden Hospital, Downs Road, Sutton, Surrey SM2 5PT, United Kingdom. Tel: 0044208661 3371; Fax: 0044 208661 3107; E-mail: john.yarnold@icr.ac.uk

Equal contribution: all authors contributed equally to this work and approved the final version.
The trial from which data were extracted was funded in part by Marks and Spencer Plc. The Institute of Cancer Research Clinical Trials and Statistics Unit and the Section of Academic Radiotherapy receive funding from Cancer Research UK.

Received Sept 2, 2009, and in revised form Oct 16, 2009. Accepted for publication Oct 16, 2009. 
relapse relative to that of the primary tumour and (ii) comparing the rates of ipsilateral NP with that in the opposite breast.

\section{METHODS AND MATERIALS}

\section{Study design}

A retrospective review was undertaken of the medical records of women with early breast cancer treated with conservative surgery and WBRT at the Royal Marsden Hospital and Gloucestershire Oncology Centre between 1986 and 1998 in the context of a prospective randomized trial of radiotherapy fractionation $(17,18)$. The original trial was approved by the local ethics committees, and this review was approved by the audit committee at the Royal Marsden NHS Foundation Trust.

\section{Original trial}

The aim of the original study was to test the effects of radiotherapy fractions of $>2$ Gy on late normal tissue responses after tumour excision and WBRT. Patients under 75 years of age at presentation with operable invasive breast cancer (T1-T3 N0-N1 M0) requiring radiotherapy were eligible for the original clinical trial if they had breast-conserving surgery and complete macroscopic resection of invasive carcinoma.

Patients were randomized to three alternative dose schedules (50 Gy in 25 fractions over 5 weeks [control], 42.9 Gy in 13 fractions five times per fortnight over 5 weeks, and 39 Gy in 15 fractions five times per fortnight over 5 weeks) at a ratio of $1: 1: 1$. Patients were stratified by treatment centre and by whether microscopic foci of invasive disease was present at or $<3 \mathrm{~mm}$ to the nearest surgical margin. Recommendations for adjuvant systemic therapy were not specified in the protocol and developed over the accrual period according to changes in routine local practice. Patient cases were reviewed at 3-month intervals for the first 3 years, 6-month intervals to 5 years, and annually thereafter. Physician assessments were prospectively recorded annually on a trial pro forma report. IBTR was recorded onto the pro forma report pictorially to show the quadrant of relapse in relation to the location of the original tumour. Histology of the relapse was also recorded on the pro forma record if available. CLBC and metastases were also recorded prospectively. Patients were followed up routinely biannually for the first 5 years with annual mammography and yearly thereafter with biennial mammography.

The primary endpoint was late change in breast appearance compared to postsurgical appearance. Secondary endpoints included palpable breast induration (fibrosis) and ipsilateral tumour recurrence.

\section{Classification of ipsilateral relapse}

For the present study, trial case report forms of all patients recorded were reviewed as having local relapse. All patients with an IBTR were identified, and a further review of clinical, mammographic, and pathology data was undertaken to classify relapses as either LR or NP. Metastatic disease at time of local relapse was also recorded. We considered only the first local relapse in patients who developed more than one ipsilateral relapse. Demographic and clinical data of each patient with an IBTR were recorded. These data included patient's age at randomization, tumour size and location at original diagnosis, nodal status at original diagnosis, histology of original tumour and of relapse, resection margin status of original tumour (complete, marginal, incomplete, or unknown) estrogen and progesterone receptor status (if available), adjuvant chemotherapy and/or hormonal therapy, location of relapse relative to primary tumour bed, and method of detection of original tumour and relapse. The location of the original tumour and the relapse was determined by review of radiotherapy records and surgical and clinical notes. Radiotherapy and follow-up data were also recorded.

After clinical and pathological data were reviewed, IBTR was classified as either NP or LR, and each category was subclassified as "definite" or "likely", depending on the strength of the collated evidence. An IBTR was classified as definite LR if it presented in the same quadrant as the original primary, determined to be the same histological type, and was the same or higher grade. IBTR was classified as definite NP if it was of a different histological type, a lower grade, and located in a different quadrant than the original primary. Tumour grade was incorporated into histological type. IBTR was classified as likely LR or likely NP based on the available evidence when any of the above data were incomplete or inconclusive. IBTR were classified as unknown if there was no information on location and histology.

The number of CLBC was recorded from the trial data, as each CLBC was recorded prospectively onto the case report form.

\section{Statistical analysis}

Descriptive statistics were used for patient and tumour characteristics. Survival analysis methods were used to analyze the events (LR, NP, and CLBC). Time to event was calculated as the time from randomization to first occurrence of the event. For the analysis of time to each event, patients who were event-free by the time of analysis or dead or lost to follow-up prior to the event were censored. In addition, for the analysis of time to NP, patients who were diagnosed with LR prior to NP were also censored as it was thought that an LR could potentially preclude or alter the probability of observing an NP. Likewise, the NP was treated as a competing risk for LR. Cumulative incidence rates with $95 \%$ confidence intervals (CI) were estimated for CLBC, using the Kaplan-Meier method, and for LR and NP, using a method accounting for the competing risk (19). Data analyses were performed using Stata version 10.1 software.

\section{RESULTS}

All 1,410 women randomized into the radiotherapy fractionation trial between 1986 and 1998 were considered for the study. Baseline characteristics have been described previously (17) and are summarized in Table 1 . The median follow-up was 10.1 years and 1,176 (99.0\%), 534 (53.4\%), and $69(9.9 \%)$ patients were still on follow-up at 5, 10, and 15 years, respectively.

There were 150 documented cases of IBTR and 71 cases of CLBC. The median age at randomization of women who developed IBTR was 50 years (interquartile range [IQR], 42-58 years). The median time to relapse from randomization was 41 months (IQR, 24-70 months). Fifty-nine (39\%) patients had metastatic disease at the time of IBTR, of whom 39/59 $(66 \%)$ patients had definite or likely LR. The classification of IBTR was as follows: definite LR, 85 (57\%) patients; likely LR, 33(22\%) patients; definite NP, 12 (8\%) patients; likely NP, 15 (10\%) patients; and unknown, 5 (3\%) patients (Table 2). The data on which 150 IBTR were classified as definite or likely, LR or NP, is illustrated in Fig. 1. Cumulative incidence rates at 5, 10, and 15 years were 6.7, 8.9, and 
Table 1. Demographic and clinical characteristics of randomized patients

\begin{tabular}{|c|c|}
\hline Patient characteristic & $\begin{array}{l}\text { Number of patients } \\
(\% \text { of } n[1,410])\end{array}$ \\
\hline \multicolumn{2}{|l|}{ Age at randomization } \\
\hline $20-29$ & $9(0.9)$ \\
\hline $30-39$ & $98(7.0)$ \\
\hline $40-49$ & $316(22.4)$ \\
\hline $50-59$ & $503(35.7)$ \\
\hline $60-69$ & $425(30.1)$ \\
\hline $70-79$ & $59(4.2)$ \\
\hline \multicolumn{2}{|l|}{ Breast size (from photographs) } \\
\hline Small & $186(13.2)$ \\
\hline Medium & $952(67.5)$ \\
\hline Large & $203(14.4)$ \\
\hline Not known & $69(4.9)$ \\
\hline \multicolumn{2}{|l|}{ Surgical deficit (from photographs) } \\
\hline Small & $845(59.9)$ \\
\hline Medium & $415(29.4)$ \\
\hline Large & $76(5.4)$ \\
\hline Not known & $74(5.2)$ \\
\hline \multicolumn{2}{|l|}{ cT stage } \\
\hline T0 & $59(4.2)$ \\
\hline $\mathrm{T} 1$ & $749(53.1)$ \\
\hline $\mathrm{T} 2$ & $575(40.8)$ \\
\hline $\mathrm{T} 3$ & $22(1.6)$ \\
\hline $\mathrm{T} 4$ & $2(0.1)$ \\
\hline $\mathrm{TX}$ & $3(0.2)$ \\
\hline \multicolumn{2}{|l|}{$\mathrm{cN}$ stage } \\
\hline No & $1,187(84.7)$ \\
\hline N1 & $219(15.5)$ \\
\hline $\mathrm{N} 2$ & $3(0.2)$ \\
\hline NX & $1(0.1)$ \\
\hline \multicolumn{2}{|l|}{ Number of nodes pathologically involved } \\
\hline 0 & $564(67.3)$ \\
\hline $1-3$ & $202(24.1)$ \\
\hline $4+$ & $72(8.6)$ \\
\hline No axillary surgery* & $572(40.6)$ \\
\hline \multicolumn{2}{|l|}{ Adjuvant treatment } \\
\hline None & $289(20.5)$ \\
\hline Tamoxifen only & $918(65.1)$ \\
\hline Chemotherapy only & $40(2.8)$ \\
\hline Tamoxifen plus chemotherapy & $156(11.1)$ \\
\hline Other & $7(0.5)$ \\
\hline \multicolumn{2}{|l|}{ Axillary/SCF treatment } \\
\hline None & $337(23.9)$ \\
\hline Axillary/SCF RT, no axillary surgery & $231(16.4)$ \\
\hline Surgery, no RT & $782(55.5)$ \\
\hline Surgery and SCF RT & $59(4.2)$ \\
\hline Not known & $1(0.1)$ \\
\hline \multicolumn{2}{|l|}{ Breast boost } \\
\hline Randomized to no boost & $359(25.5)$ \\
\hline Randomized to boost & $364(25.8)$ \\
\hline Non-randomized boost given & $687(48.7)$ \\
\hline
\end{tabular}

Abbreviations: $\mathrm{SCF}=$ supraclavicular fossa; $\mathrm{RT}=$ radiotherapy; $\mathrm{cT}=$ clinical $\mathrm{T}$ stage $; \mathrm{cN}=$ clinical node stage.

* $\mathrm{cN} 0$ and over 50 years of age.

11.8, for all LR, 0.8, 2.0, and 3.5 for all NP, and 2.4, 5.8, and 7.9 for CLBC, respectively (Table 3 ).

The median (IQR) age at randomization of women who developed CLBC was 55 (range, 45-61) years. Fifteen cases of CLBC also had IBTR (definite LR, 8 likely; LR, 4; definite NP, 1; likely NP, 2). The number of CLBC (71 cases) was at least two and a half times the number of ipsilateral NP (12 definite plus 15 likely). Even if all 33 likely LR cases were counted as NP, the total (60 cases) was still less than the number of CLBC. Figures 2 and 3 illustrate the cumulative incidence rates for definite NP, definite-plus-likely NP and CLBC. The inference is that radiation has reduced the number of ipsilateral NP tumors.

\section{DISCUSSION}

This study is based on breast-conserving surgery and WBRT associated with a 10-year Kaplan-Meier event rate of IBTR of $12 \%$, which is consistent with that of other contemporary series $(20,21)$. The number of ipsilateral NP was less than half the number of CLBC (27 compared to 71). It is not possible to explain the discrepancy in terms of a smaller volume of ipsilateral breast tissue at risk following primary surgery (where we would expect the reduction in breast tissue to be around $10 \%$ to $25 \%$ ) nor in terms of misclassification of NP. The only plausible explanation is that WBRT has a protective effect against NP tumors.

The 5-year risk of IBTR after breast-preserving surgery has previously been reported as 5 to $10 \%(15,22)$, corresponding to an annual risk of 1 to $2 \%$ (15). Several studies have attempted to differentiate between true recurrences and new primaries $(20,23-25)$, from which common themes are apparent: the majority of IBTR are LR, which tend to occur earlier, metastasize earlier and more often, and have a shorter overall and disease-free survival than NP. In our series, $59(39 \%)$ patients had metastatic disease at relapse, of whom 39/59 (66\%) patients had LR, consistent with the reported association between LR and risk of distant relapse $(21,26)$. In addition, NP is associated more often than LR in the literature with contralateral breast cancer. The median times to relapse for cases of definite and likely LR (37 and 35 months, respectively) in our series were also shorter than for definite and likely NP (86 and 63 months, respectively), in keeping with results from previous series $(20,23-25)$ and supporting our attributions of IBTR.

The number of CLBC events was $71(5 \%)$, corresponding to an annual rate of $0.56 \%(71 / 1,2766.56$ women years $\times$ 100). Although this rate is influenced by patient age and adjuvant endocrine therapies, it is comparable to published rates of $0.5 \%$ to $1 \%$ per year in other series $(27,28)$ and independent of the type of primary surgery $(29,30)$. Where under-reporting is considered a potential source of bias, CLBC is probably more likely to be under-reported in trial datasets than IBTR, so this is unlikely to account for the excess of CLBC compared to ipsilateral NP. Although adjuvant systemic therapies, including endocrine and cytotoxic therapies, reduce the incidence of new primary tumors $(14,31)$, there is no $a$ priori reason to think that this effect will be greater on the ipsilateral than on the contralateral breast. Finally, although the risk of CLBC is increased after radiotherapy, there is no reason to believe that this risk is proportionately greater than the risk of radiation-induced cancers in the ipsilateral breast. 
Table 2. Classification of 150 IBTR cases

\begin{tabular}{|c|c|c|c|c|c|}
\hline $\begin{array}{l}\text { Median age at randomization } \\
\text { (IRQ range in years) }\end{array}$ & $49(42-57)$ & $50(42-52.5)$ & $49(45-59)$ & $55(42-59)$ & $57(55-60)$ \\
\hline \multicolumn{6}{|c|}{ Histology at initial diagnosis of breast cancer } \\
\hline Ductal & 67 & 10 & 27 & 12 & 4 \\
\hline Lobular & 4 & 0 & 2 & 1 & 0 \\
\hline Comedo & 0 & 1 & 0 & 0 & 1 \\
\hline Squamous & 1 & 0 & 0 & 0 & 0 \\
\hline Unknown & 0 & 0 & 0 & 1 & 0 \\
\hline \multicolumn{6}{|c|}{ Pathological tumor stage at initial diagnosis } \\
\hline Tis & 2 & 0 & 0 & 0 & 1 \\
\hline $\mathrm{T} 1$ & 24 & 5 & 15 & 7 & 2 \\
\hline No & 65 & 9 & 28 & 12 & 4 \\
\hline N1 & 20 & 3 & 5 & 3 & 1 \\
\hline \multicolumn{6}{|l|}{ Resection margin status } \\
\hline Complete & 47 & 5 & 18 & 10 & 3 \\
\hline Marginal & 7 & 3 & 3 & 2 & 0 \\
\hline Incomplete & 22 & 2 & 9 & 3 & 1 \\
\hline Unknown & 9 & 2 & 3 & 0 & 1 \\
\hline \multicolumn{6}{|c|}{ Time from randomization to IBTR (median IRQ range in months) } \\
\hline & $37(23-52)$ & $86(53-122)$ & $35(23-70)$ & $63(43-92)$ & $37(29-54)$ \\
\hline \multicolumn{6}{|l|}{ Metastatic disease at IBTR } \\
\hline Yes & 39 & 2 & 13 & 2 & 3 \\
\hline No & 39 & 10 & 15 & 12 & 1 \\
\hline
\end{tabular}

Abbreviations: Comedo = comedogenic; Tis = Carcinoma in situ.

The data generated in this study are consistent with a recent retrospective analysis of 1,990 women with early breast cancer treated with conservative surgery and whole-breast irradiation and followed for a median 6.6 (range, 0.1-20.4) years (13). The 15-year rate of ipsilateral NP was half the rate of contralateral breast cancers (6\% [95\% CI, 3\%-9\%] versus $13 \%$ (95\% CI, $10 \%-16 \%$ ), respectively). Our rate of $3.5 \%$ (95\% CI, 2.1\%-5.4\%) for definite-plus-likely NP at 15 years was less than half the rate of CLBC (7.9\% [95\% CI, 5.9\%$10.4 \%]$ ), in keeping with that study's findings. Other studies

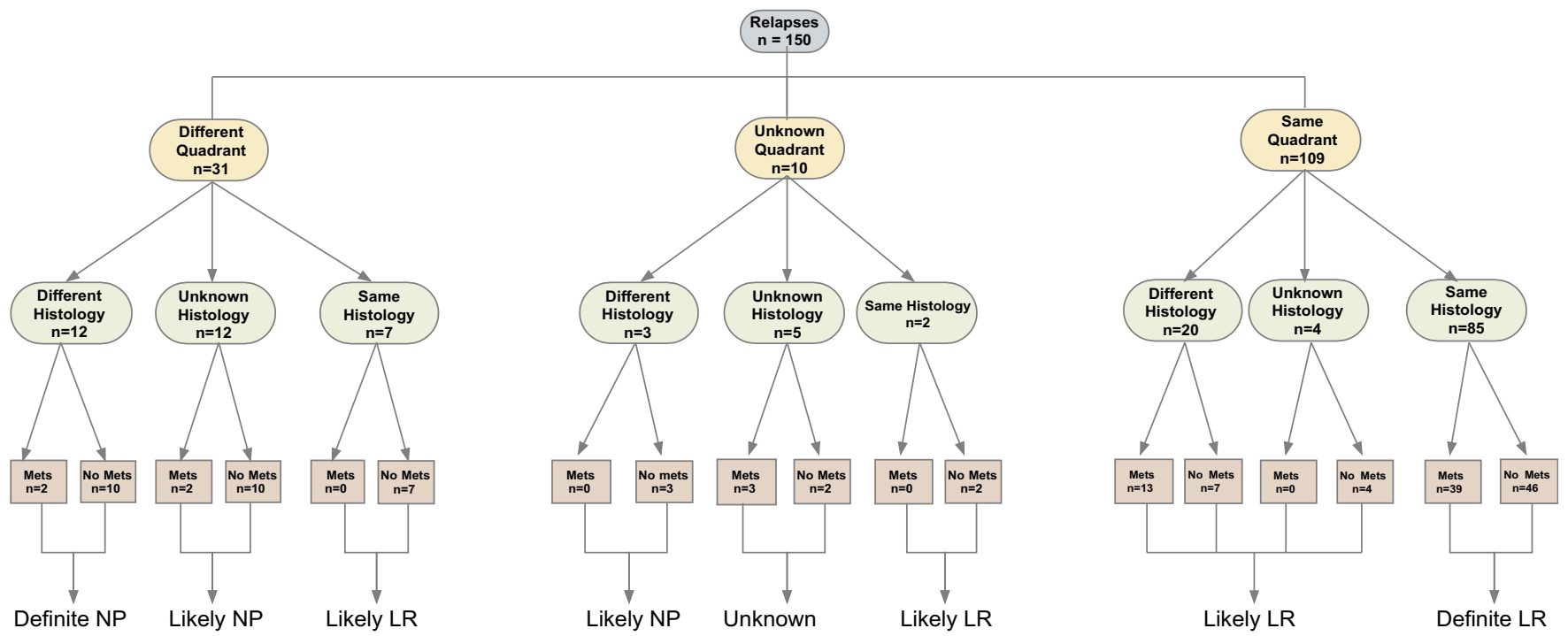

Fig. 1. Basis for classification of IBTR. 
Table 3. Cumulative incidence rates for LR, NP, and CLBC

$\%$ of incidence $(95 \% \mathrm{CI})$ at:

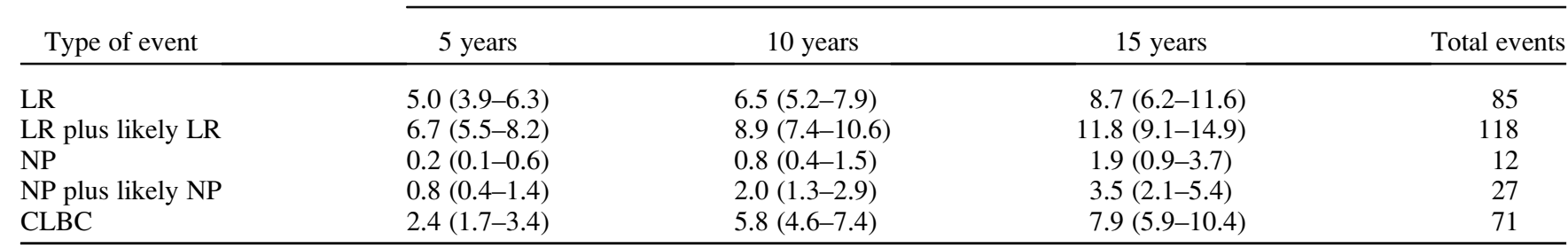

have reported similar relative rates of NP and CLBC $(20,22)$. Smith et al. (20) classified 130 cases of IBTR in 1,152 patients as NP (70) or LR (60) based on location, histology, and/or flow cytometry over a mean follow-up of 14.2 years ( \pm 0.13 years). The rates of LR and NP at 15 years were $6.8 \%$ and $13.1 \%$, respectively. The NP rate was reported as similar to the CLBC rate. However, there are important points to consider when interpreting these data. NP and CLBC rates may have been higher due to the fact that 8 of 70 IBTR cases classified as NP were BRCA1 or BRCA2 carriers, and 5 of these women developed CLBC. Importantly, all IBTR that appeared in a separate quadrant or were distinctly removed from the original primary were classified as NP. The authors acknowledged that some of these tumors may have represented multicentric disease that was not fully eradicated by radiation therapy. It is therefore likely that some NP tumors were misclassified. An important difference between that study and ours is the use of systemic treatment: in the study by Smith et al. (20), 261/1,152 and 267/1,152 patients received adjuvant chemotherapy and hormone therapy, respectively, compared with $918 / 1,410$ patients who received adjuvant hormones, 40/1,410 patients who received chemotherapy only, and $156 / 1,410$ patients who received both in our cohort. The greater number of patients who received systemic therapy in our cohort may account partly for the lower rates of NP and CLBC.

The Milan I trial (22) reported similar NP and CLBC rates in 579 women treated with quadrantectomy and randomized

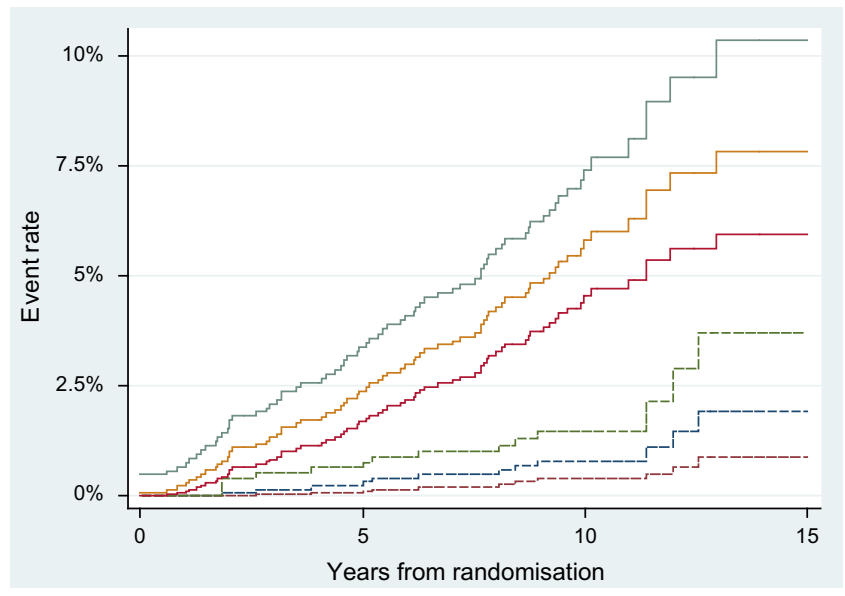

Fig. 2. Cumulative incidence rates with $95 \%$ CI for definite NP (dashed lines) versus CLBC (solid lines). to receive breast radiotherapy or not. However, the absolute numbers of NP and CLBC reported were small (10 and 22 patients, respectively). The similarity in NP and CLBC rates was noted in the group of patients treated with quadrantectomy without radiotherapy ( $n=273$ patients). In the group of women who received radiotherapy after quadrantectomy $(n=294)$, there were 2 NP events and 10 CLBC events, corresponding to $2 / 294(0.6 \%)$ and $10 / 294(3.4 \%)$ patients, respectively. This is more in keeping with our findings and consistent with a radiotherapy effect against NP. In the group not treated with radiotherapy, NP and CLBC rates were $2.9 \%$ (8/273) and 4.4\% (12/273), respectively. An earlier study by Veronesi et al. (32) reported $19 \mathrm{NP}$ and 45 CLBC events in 1,232 cases of early breast cancer treated with breast-conserving surgery and radiotherapy $(1.5 \%$ and $3.7 \%$, respectively), also in keeping with our results.

It is important to note that the analytical methods employed by these studies did not account for competing risks, which could make a difference as the competing risk event rates in their cases were higher. Our approach took into consideration competing risks, although, ultimately, a big difference compared to the Kaplan-Meier approach was not noted as the competing risk event rate was very low.

Assuming completeness of reporting IBTR and CLBC, the only way our analysis leads to a wrong conclusion is if a substantial proportion of ipsilateral LR cases have been misclassified. It would require all 33 likely LR to be reclassified as NP and added to the total of 12 definite and 15 likely NP.

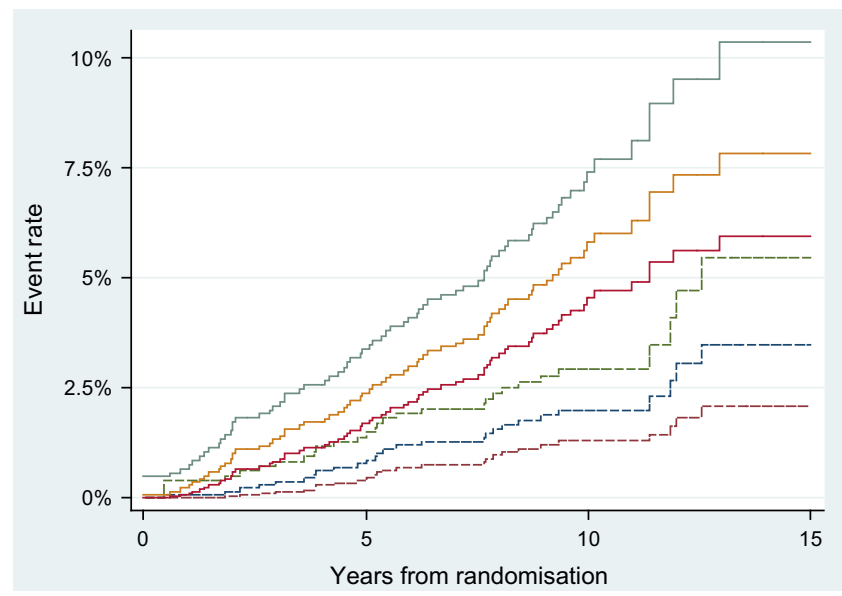

Fig. 3. Cumulative incidence rates with $95 \%$ CI for definite-pluslikely (NP + likely NP) (dashed lines) versus CLBC (solid lines). 
Even then, there would be 11 fewer NP than CLBC, a discrepancy that might be explained by the loss of breast tissue due to primary surgery and/or misclassification of a minority of definite LR. In future studies, molecular analyses may help identify the subset of IBTR that are NP on the basis of clonal differences. There have already been some clone studies looking at IBTR, ipsilateral relapses, and distant metastases and the relationships between them $(33,34)$. Meanwhile, it is fair to say that there is fairly strong evidence that what we currently classify as NP are responsive to radiotherapy.

\section{CONCLUSIONS}

This study suggests that WBRT after breast-conserving surgery for early breast cancer reduces the rate of ipsilateral NP tumors. If true, the results from this study lead to a prediction that current trials of PBRT will eventually record higher rates of IBTR after partial-breast than after whole-breast RT. Longer follow-up and robust classification of IBTR cases into LR and NP tumors are required to reflect the true rate of NP tumors.

\section{REFERENCES}

1. Veronesi U, Marubini E, Del Vecchio M, et al. Local recurrences and distant metastases after conservative breast cancer treatments: Partly independent events. J Natl Cancer Inst 1995 Jan 4;87(1):19-27.

2. Sector resection with or without postoperative radiotherapy for stage I breast cancer. a randomized trial. The Uppsala-Orebro Breast Cancer Study Group. J Natl Cancer Inst 1990;82(23): 1851.

3. Clark RM, McCulloch PB, Levine MN, et al. Randomized clinical trial to assess the effectiveness of breast irradiation following lumpectomy and axillary dissection for node-negative breast cancer. J Natl Cancer Inst 1992;84(9):683-689.

4. Wazer DE, Berle L, Graham R, et al. Preliminary results of a phase I/II study of HDR brachytherapy alone for T1/T2 breast cancer. Int J Radiat Oncol Biol Phys 2002;53(4):889-897.

5. Arthur DW, Vicini FA. Accelerated partial breast irradiation as a part of breast conservation therapy. J Clin Oncol 2005;23(8): 1726-1735.

6. Strnad V, Ott O, Potter R, et al. Interstitial brachytherapy alone after breast conserving surgery: Interim results of a GermanAustrian multicenter phase II trial. Brachytherapy 2004;3(3): 115-119.

7. Vicini FA, Arthur DW. Breast brachytherapy: North American experience. Semin Radiat Oncol 2005;15(2):108-115.

8. McCormick B. Partial-breast radiation for early staged breast cancers: hypothesis, existing data, and a planned phase III trial. J Natl Compr Canc Netw 2005;3(3):301-307.

9. Polgar C, Strnad V, Major T. Brachytherapy for partial breast irradiation: The European experience. Semin Radiat Oncol 2005; 15(2):116-122.

10. Offersen BV, Overgaard M, Kroman N, Overgaard J. Accelerated partial breast irradiation as part of breast conserving therapy of early breast carcinoma: a systematic review. Radiother Oncol 2009;90(1):1-13.

11. Orecchia R, Ciocca M, Tosi G, et al. Intraoperative electron beam radiotherapy (ELIOT) to the breast: A need for a quality assurance programme. Breast 2005;14(6):541-546.

12. Vaidya JS, Baum M, Tobias JS, et al. Targeted intra-operative radiotherapy (Targit): An innovative method of treatment for early breast cancer. Ann Oncol 2001;12(8):1075-1080.

13. Freedman GM, Anderson PR, Hanlon AL, Eisenberg DF, Nicolaou N. Pattern of local recurrence after conservative surgery and whole-breast irradiation. Int J Radiat Oncol Biol Phys 2005;61(5):1328-1336.

14. Clarke M, Collins R, Darby S, et al. Effects of radiotherapy and of differences in the extent of surgery for early breast cancer on local recurrence and 15-year survival: An overview of the randomised trials. Lancet 2005;366(9503):2087-2106.

15. Fisher B, Jeong JH, Anderson S, Bryant J, Fisher ER, Wolmark N. Twenty-five-year follow-up of a randomized trial comparing radical mastectomy, total mastectomy, and total mastectomy followed by irradiation. N Engl J Med 2002; 347(8):567-575.
16. Veronesi U, Cascinelli N, Mariani L, et al. Twenty-year followup of a randomized study comparing breast-conserving surgery with radical mastectomy for early breast cancer. $N$ Engl J Med 2002;347(16):1227-1232.

17. Yarnold J, Ashton A, Bliss J, et al. Fractionation sensitivity and dose response of late adverse effects in the breast after radiotherapy for early breast cancer: Long-term results of a randomised trial. Radiother Oncol 2005;75(1):9-17.

18. Owen JR, Ashton A, Bliss JM, et al. Effect of radiotherapy fraction size on tumour control in patients with early-stage breast cancer after local tumour excision: Long-term results of a randomised trial. Lancet Oncol 2006;7(6):467-471.

19. Vincenzo Coviello V, Boggess M. Cumulative incidence estimation in the presence of competing risks. Stata Journal 2004;4(2):103-112.

20. Smith TE, Lee D, Turner BC, Carter D, Haffty BG. True recurrence vs. new primary ipsilateral breast tumor relapse: An analysis of clinical and pathologic differences and their implications in natural history, prognoses, and therapeutic management. Int $J$ Radiat Oncol Biol Phys 2000;48(5):1281-1289.

21. Fisher B, Anderson S, Fisher ER, et al. Significance of ipsilateral breast tumour recurrence after lumpectomy. Lancet 1991; 338(8763):327-331.

22. Veronesi U, Marubini E, Mariani L, et al. Radiotherapy after breast-conserving surgery in small breast carcinoma: Longterm results of a randomized trial. Ann Oncol 2001;12(7): 997-1003.

23. Komoike Y, Akiyama F, Iino Y, et al. Analysis of ipsilateral breast tumor recurrences after breast-conserving treatment based on the classification of true recurrences and new primary tumors. Breast Cancer 2005;12(2):104-111.

24. Huang E, Buchholz TA, Meric F, et al. Classifying local disease recurrences after breast conservation therapy based on location and histology: New primary tumors have more favorable outcomes than true local disease recurrences. Cancer 2002; 95(10):2059-2067.

25. Nishimura S, Takahashi K, Akiyama F, et al. Classification of ipsilateral breast tumor recurrence after breast-conserving therapy: new primary cancer allows a good prognosis. Breast Cancer 2005;12(2):112-117.

26. Touboul E, Buffat L, Belkacemi Y, et al. Local recurrences and distant metastases after breast-conserving surgery and radiation therapy for early breast cancer. Int J Radiat Oncol Biol Phys 1999;43(1):25-38.

27. Chaudary MA, Millis RR, Hoskins EO, et al. Bilateral primary breast cancer: a prospective study of disease incidence. $\mathrm{Br} \mathrm{J}$ Surg 1984;71(9):711-714.

28. Rosen PP, Groshen S, Kinne DW, Hellman S. Contralateral breast carcinoma: An assessment of risk and prognosis in stage I (T1N0M0) and stage II (T1N1M0) patients with 20-year follow-up. Surgery 1989;106(5):904-910.

29. Fisher ER, Fisher B, Sass R, Wickerham L. Pathologic findings from the National Surgical Adjuvant Breast Project (protocol 
no. 4). XI. Bilateral breast cancer. Cancer 1984;54(12):30023011.

30. McCredie JA, Inch WR, Alderson M. Consecutive primary carcinomas of the breast. Cancer 1975;35(5):1472-1477.

31. Jonat W, Gnant M, Boccardo F, et al. Effectiveness of switching from adjuvant tamoxifen to anastrozole in postmenopausal women with hormone-sensitive early-stage breast cancer: A meta-analysis. Lancet Oncol 2006;7(12):991-996.

32. Veronesi U, Salvadori B, Luini A, et al. Conservative treatment of early breast cancer. Long-term results of 1232 cases treated with quadrantectomy, axillary dissection, and radiotherapy. Ann Surg 1990;211(3):250-259.

33. Vicini FA, Goldstein NS, Wallace M, Kestin L. Molecular evidence demonstrating local treatment failure is the source of distant metastases in some patients treated for breast cancer. Int J Radiat Oncol Biol Phys 2008;71(3):689-694.

34. Bollet MA, Servant N, Neuvial P, et al. High-resolution mapping of DNA breakpoints to define true recurrences among ipsilateral breast cancers. J Natl Cancer Inst 2008;100(1): 48-58. 